\title{
Comparison of Oocyte and Embryo Quality in Women With Polycystic Ovary Syndrome and the Control Group Candidate for In Vitro Fertilization and Intracytoplasmic Sperm Injection
}

\author{
Malihe Afiat $^{1}{ }^{\circledR}$, Nayere Khadem², Elnaz Nayeri ${ }^{3}$, Roya Jalali ${ }^{3}$, Saeed Akhlaghi ${ }^{4}$, Elahe Akhgari ${ }^{3}$, Armin \\ Attaranzadeh $^{5}$, Fateme Borzoee ${ }^{6}$, Azade Khazaie ${ }^{5}$, Behnaz Souizi ${ }^{{ }^{*} \mathbb{D}}$
}

\begin{abstract}
Objectives: Polycystic ovary syndrome (PCOS) is the most common cause of female infertility. The aim of this study was to compare the oocyte and embryo quality between the PCOS women with the control group candidate for in vitro fertilization/ intracytoplasmic sperm injection (IVF/ICSI).

Materials and Methods: The present study was designed at the Infertility Research Center of Milad in the prospective cohort format and was carried out on 100 cases of infertile women with confirmed PCOS (case group) and the male factor (control group) as the first IVF cycle candidates. Both groups underwent the ovary stimulation cycle and ICSI under the standard antagonist protocol. The collected data were then processed and analyzed using the SPSS software, version 16.

Results: The average age of study cases was $35 \pm 3$, and oocyte necrosis was the underlying pathological factor in both groups (28\% and $26 \%$ in the PCOS and control groups, respectively). In addition, most embryones belonged to either grade 1 or 2 or were 8-cell embryos. Furthermore, the highest number of transferred embryos among the patients was related to the 8-cell and grade 1. The occurrences of biochemical pregnancy in the PCOS and control groups were up to $31.91 \%$ and $22 \%$, respectively, leading to $72.73 \%$ and $60 \%$ childbirth in cases of both groups. Finally, there were no significant differences observed with respect to the quality and the quantity of the embryones, the oocyte, the transferred embryo, the germinal vesicle oocytes, and the rate of pregnancy among the two groups $(P>0.05)$.

Conclusions: According to the results of the present study, no differences were found concerning the oocyte quality, embryo, and the pregnancy rate between PCOS cases and any other patients requiring ICSI. Therefore, such cases can similarly benefit from ICSI methods as well.

Keywords: Polycystic ovary syndrome, Oocyte, Embryo, Intracytoplasmic sperm injection
\end{abstract}

\section{Introduction}

Polycystic ovary syndrome (PCOS) is the most prevalent endocrinopathy and one of the most common causes for female infertility found in $6.5 \%$ to $8 \%$ of the worldwide women population (1).

Controlled ovarian hyperstimulation as an assisted reproductive technology (ART) involves the use of some fertility medications to induce ovulation. Moreover, intracytoplasmic sperm injection (ICSI) is an in vitro fertilization (IVF) method in which a single sperm cell is directly injected into the cytoplasm of an ovum. ICSI is used to provide the necessary gametes for the obtainment of embryos that may be transmitted to the uterus cavity (2).

The primary oocyte quality has an utmost important effect on the survival of the embryo, as well as in the establishment and maintenance of pregnancy, fetus growth, and even in the possible adulthood illnesses (3).

Additionally, oocyte quality in cases with PCOS can be afflicted due to the inapt signaling between the cellular accumulation and oocyte (3). Furthermore, abnormalities such as endocrine/paracrine, metabolic activity disorder, and intrafollicular changes during the folliculogenesis and follicle maturation can lead to incomplete maturation of the embryo and its ability to grow in PCOS patients. Lowquality oocyte cells in such cases can reduce the pregnancy rate, the splitting, and implantation, and thus increase the likelihood of miscarriage (4).

Given that the PCOS is believed to be a leading factor in the deterioration of the oocyte quality, such a syndrome can be named to be the main cause of female infertility. 


\section{Key Message}

- PCOS as the main cause of female infertility is a leading factor in the deterioration of the oocyte quality.

- Based on this study, the oocyte and the transferred embryos quality in PCOS patients were similar to control group.

Considering that no similar study is available regarding the effect of PCOS on oocyte and embryo quality and fertility prognosis following the IVF procedure in Iran, this study was conducted to determine a comparison of the quality of the oocyte and the embryo between the infertile women with PCOS and control group candidates for IVF/ICSI.

\section{Materials and Methods}

This study followed a cohort and prospective format and was carried out in the Milad Infertility Research Center affiliated with Mashhad University of Medical Sciences. The cases were all infertile women candidates for the first IVF cycle. In addition, the study was executed after acquiring the ethics code of 940581 from the Ethics Committee of Mashhad University of Medical Sciences during 2015-2017. The couples who entered the IVF cycle program were given a complete explanation of the purpose of the study, followed by obtaining written consent in case the couples agreed to participate in the study. The demographic specifications of the subjects were recorded, and then they were divided into two groups.

\section{Participants}

The case group consisted of 50 women with the confirmed diagnosis of PCOS who were also undergoing ICSI. These patients had no spermiogram-based male factor and were resistant to clomiphene treatment. In the control group, 50 infertile women were undergoing ICSI based on male factor indication. The women in this group had regular menstruation with no signs of hyperandrogenism and they were of $10>$ follicle-stimulating hormone (FSH). The inclusion criteria for both groups were being in the age range of 20-30 years, having a body mass index of less than $32 \mathrm{~kg} / \mathrm{m}^{2}$, having no underlying disorders, and being non-smokers and non-substance users. On the other hand, individuals were excluded from the study in case there was a history of uterine anomaly or uterine surgery, endometriosis grades 3 and 4, or the presence of hydrosalpinx in the sonography. In addition, the exclusion criteria consisted of cases in which a non-responsive ovary led to the cancellation of the cycle, hyperstimulation was observed, or those in which human chorionic gonadotropin (HCG) was not injected, or the times when the patient did not refer back for treatment.

Based on Rotterdam Criteria (5), the diagnosis criteria for PCOS were having 2 out of 3 following cases:
1. Oligomenorrhea, 2. Clinical symptoms or laboratory findings of hyperandrogenism, and 3. Observation of PCO in the sonography of ovaries, that is, the existence of 12 or more $2 \sim 9 \mathrm{~mm}$ antral follicles (6).

\section{Clinical Evaluation}

Stimulation Protocol

Under the standard antagonist protocol, patients assigned to both study groups entered into the control ovarian stimulation cycle. Ovarian stimulation was initiated when pituitary desensitization was achieved (the absence of follicle diameter $>10 \mathrm{~mm}$ and the estradiol level of $<40$ $\mathrm{pg} / \mathrm{mL}$ ) using $150 \mathrm{IU}$ (7) of recombinant human FSH (rhFSH, Gonal-F ${ }^{\circledR}$, Merck Serono SA, Switzerland). The first follicular growth was monitored via transvaginal ultrasonography (8) on the 6th day of the cycle. Then, rhFSH was administered in a step-up fashion and the dose of rhFSH was adjusted every 2 to 3 days or HMG (Meropur ${ }^{\circledR}$, Ferring Pharmaceuticals, Germany or Merional ${ }^{\circledR}$, IBSA, Switzerland) was added if necessary according to ovarian response in sonography (9). The follicular growth was constantly monitored via transvaginal ultrasonography every 2 to 3 days. When at least 1 follicle reached 13-14 $\mathrm{mm}, 0.25 \mathrm{mg}$ of gonadotropin-releasing hormone ( $\mathrm{GnRH})$ antagonist (Cetrorelix acetate, Cetrotide, Merck Serono SA, Switzerland) (10) was started and continued until HCG administration. Estradiol was measured as soon as at least 3 follicles with over 18 millimeters of diameter were observed to specify the underlying type of the trigger. In the case of the serum estradiol level more than $5000 \mathrm{pg} /$ $\mathrm{mL}$ (10) on the day, the GnRH agonist was injected so that to avoid the risk of ovarian hyperstimulation syndrome. The patient was then removed from the study. Otherwise, HCG in the amount of 10.000 IU (Choriomon, IBSA, Switzerland) (11) was injected to serve as the trigger so that to complete the final stage of oocyte maturation.

\section{Oocyte Retrieval}

Transvaginal ultrasound (8) guided oocyte collection was performed 36 hours after HCG injection. The obtained oocyte was initially incubated in the fertilization medium (ORIGIO $^{\circledR}$ Sequential Cleav ${ }^{\mathrm{TM}}$, Denmark) at 37 centigrade temperature with $6 \% \mathrm{CO}_{2}$ and $5 \% \mathrm{O}_{2}$ for a minimum of 2 hours. Next, the oocyte was identified in the horizontal laminar flow hood with the Olympus stereo microscope, and the oocyte was clearly visible. Then, oocytes were denudated from cumulus oophorus by exposure to an $80 \mathrm{IU} / \mathrm{mL}$ hyaluronidase enzyme (12) in the HEPESbuffered medium, followed by the mechanical removal of the corona radiata. In addition, oocytes were assessed for their maturation and morphology under an inverted microscope. Good-quality mature human oocytes have a clear, moderately granular, homogeneous cytoplasm that does not contain inclusions (a small perivitelline space, a round clear, colorless zona pellucida, and single unfragmented polar body). Further, dysmorphic oocytes 
are commonly classified as having abnormal cytoplasm (dark cytoplasm, not homogenous, or increased granularity), cytoplasmic inclusions (vacuoles and refractile bodies), an abnormal oocyte shape (oval), an abnormal zona pellucida, an abnormal perivitelline space, and an abnormal polar body.

\section{Sperm Preparation}

On the morning of oocyte retrieval, the semen was prepared from the patient's spouse, and then sperm washing was done in the andrology laboratory after initial examination based on the patient's condition using the Zetta, Ericsson, or swim-up method. After sperm preparation, the sample was delivered to the ART laboratory for injection into the oocyte.

\section{Fertilization}

Finally, the ICSI was carried out after 2 hours of oocyte incubation using a special needle through advanced microscopes.

The fertilized oocytes were then placed in an incubator at $37^{\circ} \mathrm{C}$ and multigas for 3 days. This process took place 2 to 5 hours after the ICSI. Eventually, embryos were cultivated using a sequential media approach and were daily monitored to ensure the achieved quality.

\section{Embryo Transfer}

The transfer was guided by abdominal ultrasound with a full bladder, and soft catheters were used for embryo transfer (Soft-Pass ${ }^{\mathrm{TM}}$ Embryo Transfer Catheter Set, Cook Medical). The embryos (1 or 2) were chosen for transfer depending on their quality and transferred to the uterus after 2, 3, or 5 days. Luteal phase support started from the day of oocyte pick-up using the $600 \mathrm{mg}$ (13) of Utrogestan Vaginal. The beta-human chorionic gonadotrophin ( $\beta \mathrm{hCG}$ ) was ultimately measured 14 days since the day of transfer.

\section{Outcomes}

The outcome examination included the quantity and the quality of the oocytes, as well as the number and the quality of the resulting embryos, chemical pregnancy (the appearance of $\beta \mathrm{hCG}$ in the blood 2 weeks after the transfer), the occurrence of miscarriages (the loss of pregnancy before 20 weeks), and the number of live childbirths (the ratio number of pregnancies leading to a live baby born as per each patient). Next, the oocyte quality was determined using optical microscopes by a pathological technician who was unaware of subject categorization. Based on pathological findings, the quality of the picked-up oocytes from the patients of both groups was classified into metaphase 2 oocytes (first candidates for injection), metaphase 1, and Grade 3 (incapable of being injected or injection in the case of maturation). The quality of the embryos was assessed based on pathological criteria in grades I, II, and III (14).
Grade I: The embryos being of the same size blastomeres and 20\% lesser than granulation and fermentation;

Grade II: The embryos being of unequal sizes and between $20 \%$ and $40 \%$ of granulation and fermentation;

Grade III: The embryos being of unequal sizes and over $40 \%$ granulation and fermentation.

Sample Size

According to the protocol of the study by Einsler et al (15), where the number of oocytes was $15.3 \pm 5.3$ and $11.7 \pm 6.1$ in the PCOS and control groups, the sample size (with the power of $80 \%$ and the $95 \%$ factory of safety) was obtained through division into two domains based on the comparison of means equal to 50 individuals in each group.

\section{Data Analysis}

All continuous and categorical variables were expressed as mean \pm standard deviation (SD) and frequency (\%), respectively, and the normality of variables was checked by the Kolmogorov-Smirnov test. In addition, the oocyte quality and transferred embryo quality were compared among the groups using the chi-square test. Further, Fisher's exact test was used to compare clinical pregnancy and live birth between the two groups. Finally, data analysis was performed in SPSS, version 16, and the level of 0.05 was considered statistically significant.

\section{Results}

The average age of participants was $34 \pm 3$ and $36 \pm 5$ years of age and in the PCOS and control groups, respectively, and the average duration of infertility in both groups was 24 months. Moreover, the average body mass index in the PCOS and control groups was 26 and $23.4 \mathrm{~kg} / \mathrm{m}^{2}$, respectively.

The quality evaluation of the picked-up oocytes from the patients in both groups is presented in Table 1. As shown, most aspirated oocytes were in the highest quality (metaphase II), which was similar in PCOS and control patients. Ovum necrosis was the most common pathology among the two groups. Based on the results of chi-square and Fisher' exact tests, no significant differences were found with regard to the observed oocyte quality between the two groups $(P>0.05)$.

Next, the quality of the transferred embryos was assessed in both PCOS and control groups. As depicted in Figure 1 , the majority of the embryos in both groups were from Grades 1 and 2. The results of the Chi-square test did not reveal a significant difference in the embryo quality grade between the two groups $(P=0.620)$.

Figure 2 displays the results related to the quality evaluation of the entire quantity of the resulting embryos based on the number of cells in both groups. Based on the results, the highest embryo frequency in terms of the number of cells in the PCOS and control groups was related to the 8 -cell ones which recorded 40 and 39 cases, 
Table 1. Evaluation of Oocyte Quality Between PCOS and Control Groups

\begin{tabular}{|c|c|c|c|c|c|c|c|}
\hline \multirow{3}{*}{ Oocyte Quality } & & \multirow{3}{*}{ Total (N) } & \multicolumn{5}{|c|}{ Group } \\
\hline & & & \multicolumn{2}{|c|}{ PCOS } & \multicolumn{2}{|c|}{ Control } & \multirow{2}{*}{$P$ Value } \\
\hline & & & $\mathbf{N}$ & Mean \pm SD & $\mathbf{N}$ & Mean \pm SD & \\
\hline Metaphase II & - & 100 & 50 & 8.3 & 50 & 7.7 & $0.432^{\mathrm{a}}$ \\
\hline \multirow{2}{*}{ Metaphase I } & Vacuolar & 6 & 3 & 1 & 3 & 1 & $0.634^{\mathrm{b}}$ \\
\hline & Granular & 1 & 0 & - & 1 & 1 & $0.543^{b}$ \\
\hline \multirow{2}{*}{ Grade III } & Necrosis & 27 & 14 & $3.36 \pm 2.17$ & 13 & $2.15 \pm 1.46$ & $0.422^{\mathrm{a}}$ \\
\hline & Germinal vesicle & 14 & 8 & $1.5 \pm 1.07$ & 6 & $2.0 \pm 1.10$ & $0.315^{\mathrm{a}}$ \\
\hline
\end{tabular}

Note. SD: Standard deviation; PCOS, Polycystic ovary syndrome; ${ }^{\text {b }}$ Chi-square test; ${ }^{\text {b }}$ Fisher exact test.

respectively. The chi-square test showed no significant difference with regard to this finding between the two groups $(P=0.511)$.

Moreover, the quality of the transferred embryo in terms of the grade and the number of cells was assessed following the description of the overall quality of the embryos. The task was carried out on the day of embryo transfer in both groups (Table 2). The Chi-square results demonstrated no significant difference between the two groups in this respect $(P=0.620)$. The results further represented that the highest number of transferred embryos in the two

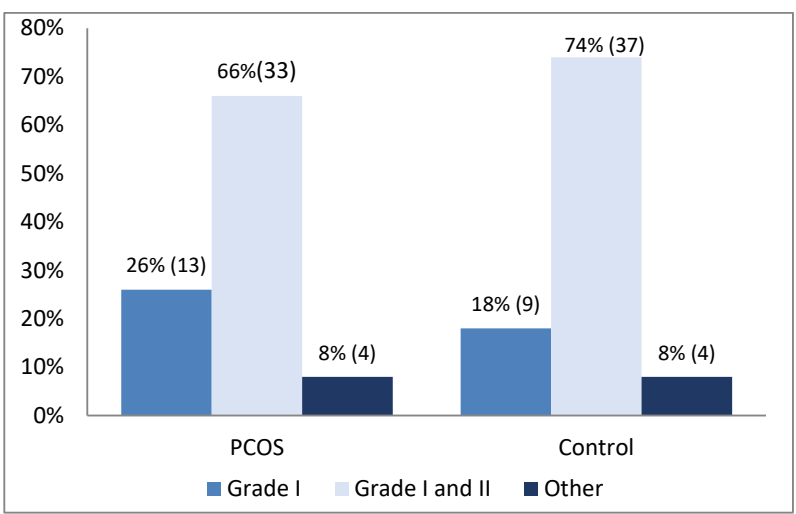

Figure 1. The Quality of Embryos in Terms of Grade Between PCOS and Control Groups. Note. PCOS: Polycystic ovary syndrome.

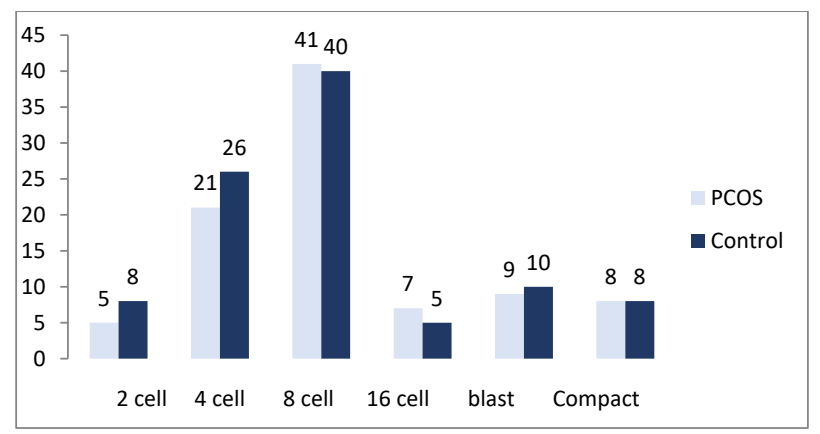

Figure 2. The Overall Quality of Embryos in Terms of the Number of Cell Grade Between PCOS and Control Groups. Note. PCOS: Polycystic ovary syndrome. groups belonged to the 8-cell ones (37 and 39 cases from PCOS and control groups, respectively). Furthermore, among all the 8-cell embryos from the PCOS and control groups, most cases belonged to Grade 1 rather than any other grade (24 and 28, respectively). A series of other information is presented in Table 2. Figure 3 provides a comparison in terms of assessing the rate of chemical pregnancy occurrences based on the positive $\beta$ hCG in patients of both PCOS and control groups. Although the number of clinical pregnancies happening in the PCOS group was slightly more than that of the Control group (31.91\% and 22\%, respectively), the Fisher's exact test showed no significant difference in this regard $(P=0.361)$.

The patients from both groups were re-examined as a follow-up procedure for the sake of the final evaluation of pregnancy results 14 days after the embryo transfer with positive $\beta$ hCG. Based on the data in Figure 4, eight out of 11 cases from the control group (72.73\%) who underwent chemical pregnancy gave birth. Additionally, 9 out of 15 (60\%) patients, who were assigned to the PCOS group and underwent chemical pregnancy, were reported to give live birth. Eventually, Fisher's exact test did not suggest any significant difference between the PCOS and control group in terms of the comparison of the live childbirth $(P=0.683)$.

\section{Discussion}

This study was carried out for the purpose of oocyte and embryo quality assessment after using the controlled ovarian stimulation technique in infertile women with PCOS who were candidates for ICSI and to compare these results with the control group. The results of the study represented no significant difference between PCOS and control groups in terms of the number of oocyte and the number of embryones. Concerning the number of transferred Grade I embroyenes distinguished for the quality when compared to other grades, no significant differences were observed between the two groups.

Among other findings obtained through the course of this study, the rate of chemically induced pregnancy can be mentioned based on which, the positive $\beta$ hCG was reported 14 days after embryo transfer. A similar 
Table 2. Evaluation of Embryo Transfer Between PCOS and Control Groups

\begin{tabular}{|c|c|c|c|c|c|}
\hline \multirow{3}{*}{\multicolumn{2}{|c|}{ Embryo Quality/ Embryo Grading }} & \multicolumn{4}{|c|}{ Group } \\
\hline & & \multicolumn{2}{|c|}{ PCOS } & \multicolumn{2}{|c|}{ Control } \\
\hline & & No. (\%) & Mean \pm SD & No. (\%) & Mean \pm SD \\
\hline \multirow{2}{*}{ 4-cell } & Grade I & 4 & $1.5 \pm 1$ & 7 & $2 \pm 0.82$ \\
\hline & Grade II & 0 & - & 4 & 1 \\
\hline \multirow{3}{*}{ 8-cell } & Grade I & 24 & $1.75 \pm 0.85$ & 28 & $1.82 \pm 0.86$ \\
\hline & Grade II & 12 & $1.17 \pm 0.58$ & 11 & 1 \\
\hline & Grade III & 1 & 2 & 0 & - \\
\hline \multirow{2}{*}{ 16-cell } & Grade I & 7 & $1.29 \pm 0.49$ & 2 & 1 \\
\hline & Grade II & 6 & 1 & 5 & $1.2 \pm 0.45$ \\
\hline \multirow{2}{*}{ Blast } & Grade I & 12 & $1.08 \pm 0.29$ & 10 & $1.4 \pm 0.52$ \\
\hline & Grade II & 3 & $1.33 \pm 0.58$ & 6 & 1 \\
\hline \multirow{2}{*}{ Compact } & Grade I & 8 & $1.38 \pm 0.52$ & 5 & $1.4 \pm 0.55$ \\
\hline & Grade II & 0 & - & 2 & 1 \\
\hline
\end{tabular}

Note. SD: Standard deviation; PCOS, Polycystic ovary syndrome.

observation was made in patients from both groups. Additionally, the rate of clinical pregnancy revealed no significant difference between the two groups. In a study carried out by Costello et al, 135 patients with natural ovary status and 65 patients suffering from PCOS were surveyed for IVF/ICSI (16). Based on their results, no significant differences were found between the two groups

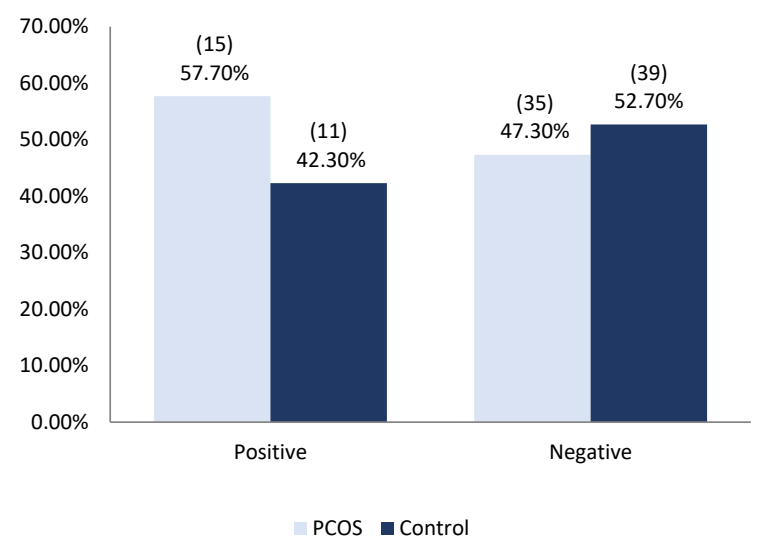

Figure 3. The Comparison Rate of Chemical Pregnancy Occurring in PCOS and Control Groups. Note. PCOS: Polycystic ovary syndrome.

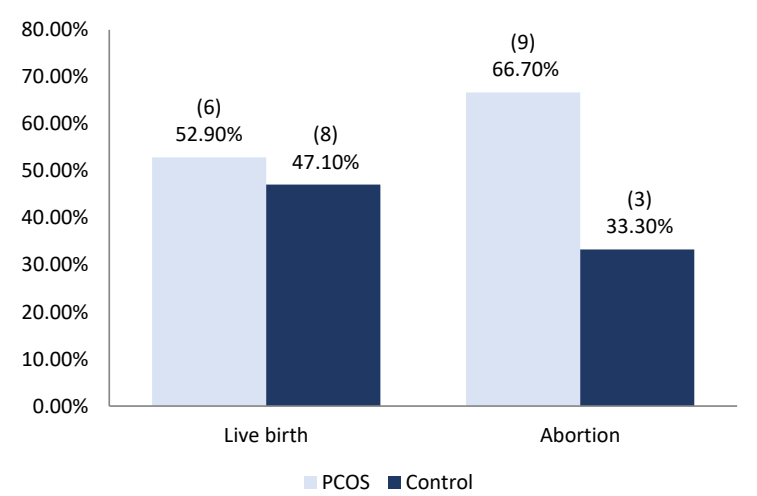

Figure 4. The Comparison of Clinical Pregnancy Results in PCOS and Control Groups. Note. PCOS: Polycystic ovary syndrome. in terms of the rate of live childbirth or the biochemical pregnancy or clinical as per each patient, which is in line with the results of the present study.

In another study, Sigala et al reported that the overall number of the oocyte and the aspirated ones in the Metaphase II, along with the number of the embryones was higher in the PCOS group (17), which contradicts the findings of the present study. No significant difference was observed between the two groups of participant patients in this study. Although the percentages of high-quality embryones on the third day were similar in both groups in the aforementioned study, the rate of induction and pregnancy in the PCOS was higher. Nevertheless, in the present study, no significant differences were observed in terms of the pregnancy rate in both groups. Eventually, Sigala et al (17) concluded that the ovarian morphology in the polycystic cases has no severely negative effect on the quality of oocytes and the embryos and it is not a leading factor resulting in ICSI, which corroborates with the results of the current study.

Contrary to the present study, the findings of Esinler et al revealed that the mean total of Grade I embryos in the PCOS group was higher when compared to the Control group. However, similar to this study, the mean total of the transferred embryos from among the three groups represented no significant difference (15).

Based on the findings of the present study, several disorders were evident in the quality of oocytes in all patients while no significant differences were found between the PCOS and control groups in terms of the type of such disorders or the number of disorders. Additionally, no significant differences were observed between the PCOS and control groups with respect to the embryo quality based on the assessment of the grades and the number of the cells.

Likewise, Sahu et al assessed the oocyte quality in women in PCOS and control groups in which the male factor was also a necessity for the ICSI procedure. It was discovered that a larger number of oocytes can be extracted in PCOS 
and PCO groups. However, embryos with 2 pronuclei were similarly prevalent in all three groups. Furthermore, no significant differences were reported regarding oocyte maturation, oocyte dysmorphism, embryo quality, and the pregnancy rate between the three groups (18). These results are in conformity with those of the present study although the results of this study indicated no significant difference with regard to the number of oocytes between the two groups.

According to the report by Ludwig et al (19), the cytoplasmic factor could result in negative outcomes in ICSI carried out for PCOS cases. Nevertheless, Sahu et al (18) focused on the likeness in oocyte morphology during the Metaphase II in PCOS and control group women, which is in line with the findings of the present study representing no significant differences between the two groups in terms of the oocyte, embryo quality, and the pregnancy rate.

The findings of the present study also showed that the chemical and clinical pregnancy rates per transfer cycle were higher in the PCOS group, and the odds ratio and the live birth rate were comparable. In addition, a higher miscarriage rate was observed in the PCOS group although this did not reach a statistically significant level. This meta-analysis demonstrated that PCOS patients may have more favourable ICSI outcomes compared to non-PCOS patients. Nonetheless, our findings should be evaluated in further prospective cohort studies.

The comparison of clinical pregnancy occurrences based on the positive $\beta$ hCG in patients demonstrated that the number of clinical pregnancies happening in the PCOS group was slightly more than that of the control group. Although the results were comparable, there were no statistically significant differences. Pregnancy outcomes were followed up as well. Most patients in PCOS and control groups had the childbearing experience, but this difference was not statistically significant. In another meta-analysis study, Duan et al reported that the clinical pregnancy rate was higher in the PCOS group and the live birth rate was comparable with the control group (20).

One of the strongest points of the present study is that it was carried out in the prospective format. Further, although there were only a few studies assessing the comparison of Grade I embryones in the two groups, the present study paid special attention to the matter.

The present research aimed only at evaluating the morphological complications of the oocyte, which is one of its limitations. A more comprehensive result can be achieved through using biochemical methods in order to measure the serum levels of a number of hormones such as follicle-stimulating hormone, luteinizing hormone, progesterone, estradiol, and the like. Additionally, the small sample size in the present study is another limitation which limits the potential for generalization.

It should be noted that the present study mainly sought to assess the quality of oocytes and the embryos in PCOS cases when compared to the control group. Notwithstanding the above-mentioned fact, the analysis of severe complications ensuing the ICSI procedure (e.g., ovarian hyperstimulation syndrome among the patients from both groups) could serve as a factor in the comprehensiveness of the results. Accordingly, it is suggested that future studies consider the complications in PCOS patients under ICSI treatment. It is also recommended that such studies evaluate and compare the rate of successful pregnancies, oocyte quality, and embryo quality, and the biochemical variables involved in the pregnancy process of PCOS patients or those suffering from any other causes of infertility.

\section{Conclusions}

In summary, the findings of the present study revealed that there were no significant differences in the number of oocytes and the embryos between PCOS and control groups. Furthermore, the oocyte quality and the quality of the transferred embryos in both groups were similar. On the other hand, the results from ICSI in PCOS patients and the control group were similar as well. Additionally, the comparison of the rate of biochemical and clinical pregnancies between the two groups represented no significant difference.

In the other words, PCOS patients can benefit from ICSI treatment and obtain similar results in terms of pregnancy rates compared to other infertile patients.

\begin{abstract}
Authors' Contribution
MA participated in conception of the design, conducting the study and revising the draft, $\mathrm{NKH}$ contributed to the data collection. EN and RJ contributed to the data collection and drafting the work. SA contributed to the analysis of the data, revising the draft. EA contributed to the data collection, approval of the final version of the manuscript, and agreed for all aspects of the work. AA contributed to conducting the study. $\mathrm{FB}$ contributed to the drafting the work, revising it and interpretation of data. BS contributed to the conception of the design, drafting the work and revising it critically for important intellectual content. ZKH contributed to the data collection. All authors approved the final version of the manuscript and agreed for all aspects of the work.
\end{abstract}

\section{Conflict of Interests}

Authors declare that they have no conflict of interests.

\section{Ethical Issues}

This study was approved by the Regional Ethics Committee of Mashhad University of Medical Sciences under the code number of T4442.1395.20.

\section{Financial Support}

The Deputy of Research and Technology in the Medical Faculty of Mashhad University of Medical Sciences provided financial support for conducting the present study under the research number of 940581 .

\section{Acknowledgments}

We have been honored and humbled to have the opportunity to contribute this article to the Milad Infertility Center, Mashhad University of Medical Sciences.

\section{References}

1. Varadan M, Gopalkrishna P, Bhat PV, et al. Influence of polycystic ovary syndrome on the periodontal health of Indian women 
visiting a secondary health care centre. Clin Oral Investig. 2019;23(8):3249-3255. doi:10.1007/s00784-018-2741-2

2. Kwan I, Wang R, Pearce E, Bhattacharya S. Pain relief for women undergoing oocyte retrieval for assisted reproduction. Cochrane Database Syst Rev. 2018;5(5):CD004829. doi:10.1002/14651858. CD004829.pub4

3. Decanter C. Oocyte quality in PCOS. In: Infertility in Women with Polycystic Ovary Syndrome. Springer; 2018:31-39. doi:10.1007/978-3-319-45534-1_4

4. Bu Z, Hu L, Su Y, Guo Y, Zhai J, Sun YP. Factors related to early spontaneous miscarriage during IVF/ICSI treatment: an analysis of 21,485 clinical pregnancies. Reprod Biomed Online. 2020;40(2):201-206. doi:10.1016/j.rbmo.2019.11.001

5. Koltun KJ, Williams NI, Scheid JL, De Souza MJ. Discriminating hypothalamic oligomenorrhea/amenorrhea from hyperandrogenic oligomenorrhea/amenorrhea in exercising women. Appl Physiol Nutr Metab. 2020;45(7):707-714. doi:10.1139/apnm-2019-0640

6. Goetsch AL, Kimelman D, Woodruff TK. Polycystic ovary syndrome. In: Fertility Preservation and Restoration for Patients with Complex Medical Conditions. Springer; 2017:231-248.

7. Bagchus W, Yalkinoglu Ö, Wolna P. Open-Label, Randomized, Two-Way, Crossover Study Assessing the Bioequivalence of the Liquid Formulation versus the Freeze-Dried Formulation of Recombinant Human FSH and Recombinant Human LH in a Fixed 2:1 Combination (Pergoveris $\left({ }^{\circledR}\right)$ ) in Pituitary-Suppressed Healthy Women. Front Endocrinol (Lausanne). 2017;8:371. doi:10.3389/ fendo.2017.00371

8. Blankstein J, Aziz P, Malepati S, Amir J. Ultrasound in follicle monitoring for ovulation induction/IUI. In: Stadtmauer L, TurKaspa I, eds. Ultrasound Imaging in Reproductive Medicine. Cham: Springer; 2019:249-271. doi:10.1007/978-3-030-166991_15

9. Kawai K, Ishikawa T, Ohuchi K, et al. WHO Group I ovulation disorder successfully treated with recombinant follicle stimulating hormone: a revisit to the roles of luteinizing hormone and estradiol. J Med Dent Sci. 2018;65(2):107-112. doi:10.11480/jmds.650208

10. Zeng C, Shang J, Jin AM, Wu PL, Li X, Xue Q. The effect of luteal $\mathrm{GnRH}$ antagonist on moderate and severe early ovarian hyperstimulation syndrome during in vitro fertilization treatment: a prospective cohort study. Arch Gynecol Obstet. 2019;300(1):223233. doi:10.1007/s00404-019-05163-3
11. Salama M, Hamza $H$. Drospirenone containing combined oral contraceptive premedication before letrozole ovulation induction in clomiphene resistant PCOS, is it worth the wait? Gynecol Reprod Health. 2019;3(2):1-6.

12. Rienzi LF, Maggiulli R, Ubaldi FM. Oocyte denuding. In: Nagy Z, Varghese A, Agarwal A, eds. In Vitro Fertilization. Cham: Springer; 2019:133-145. doi:10.1007/978-3-319-43011-9_14

13. Child T, Leonard SA, Evans JS, Lass A. Systematic review of the clinical efficacy of vaginal progesterone for luteal phase support in assisted reproductive technology cycles. Reprod Biomed Online. 2018;36(6):630-645. doi:10.1016/j.rbmo.2018.02.001

14. Hamamah S, El Messaoudi S, Thierry A, Assou S. Methods for Determining the Quality of an Embryo. Google Patents; 2019.

15. Esinler I, Bayar U, Bozdag G, Yarali H. Outcome of intracytoplasmic sperm injection in patients with polycystic ovary syndrome or isolated polycystic ovaries. Fertil Steril. 2005;84(4):932-937. doi:10.1016/j.fertnstert.2005.04.028

16. Costello MF, Chew CYM, Lindsay K, Wang A, McNally G. Effect of polycystic ovaries on in vitro fertilization and intra-cytoplasmic sperm injection treatment outcome. Asian Pac J Reprod. 2016;5(3):182-187. doi:10.1016/j.apjr.2016.04.011

17. Sigala J, Sifer C, Dewailly D, et al. Is polycystic ovarian morphology related to a poor oocyte quality after controlled ovarian hyperstimulation for intracytoplasmic sperm injection? results from a prospective, comparative study. Fertil Steril. 2015;103(1):112-118. doi:10.1016/j.fertnstert.2014.09.040

18. Sahu B, Ozturk O, Ranierri M, Serhal P. Comparison of oocyte quality and intracytoplasmic sperm injection outcome in women with isolated polycystic ovaries or polycystic ovarian syndrome. Arch Gynecol Obstet. 2008;277(3):239-244. doi:10.1007/ s00404-007-0462-x

19. Ludwig M, Finas D, Al-Hasani S, Diedrich K, Ortmann O. Oocyte quality and treatment outcome in intracytoplasmic sperm injection cycles of polycystic ovarian syndrome patients. Hum Reprod. 1999;14(2):354-8.

20. Duan N, Wang H, Niu Z, et al. A meta-analysis of oocyte quality and clinical outcome of intracytoplasmic sperm injection cycles in patients with polycystic ovary syndrome. J Med Imaging Health Inform. 2018;8(9):1886-1894. doi:10.1166/jmihi.2018.2519

(C) 2021 The Author(s); This is an open-access article distributed under the terms of the Creative Commons Attribution License (http:// creativecommons.org/licenses/by/4.0), which permits unrestricted use, distribution, and reproduction in any medium, provided the original work is properly cited. 